\title{
Interchain interaction and fractionally charged solitons in a commensurate charge- density-wave system
}

Jensen, Mogens Høgh; Lomdahl, P. S.

Published in:

Physical Review B

Link to article, DOI:

10.1103/PhysRevB.26.1086

Publication date:

1982

Document Version

Publisher's PDF, also known as Version of record

Link back to DTU Orbit

Citation (APA):

Jensen, M. H., \& Lomdahl, P. S. (1982). Interchain interaction and fractionally charged solitons in a commensurate charge-density-wave system. Physical Review B, 26(2), 1086-1089.

https://doi.org/10.1103/PhysRevB.26.1086

\section{General rights}

Copyright and moral rights for the publications made accessible in the public portal are retained by the authors and/or other copyright owners and it is a condition of accessing publications that users recognise and abide by the legal requirements associated with these rights.

- Users may download and print one copy of any publication from the public portal for the purpose of private study or research.

- You may not further distribute the material or use it for any profit-making activity or commercial gain

- You may freely distribute the URL identifying the publication in the public portal 


\title{
Interchain interaction and fractionally charged solitons in a commensurate charge-density-wave system
}

\author{
M. Høgh Jensen \\ Laboratory of Applied Mathematical Physics, The Technical University of Denmark, DK-2800 Lyngby, Denmark \\ and H.C. Orsted Institute, University of Copenhagen, ${ }^{*}$ DK-2100 Copenhagen, Denmark \\ P. S. Lomdahl ${ }^{\dagger}$ \\ Laboratory of Applied Mathematical Physics, The Technical University of Denmark, DK-2800 Lyngby, Denmark
}

(Received 14 April 1982)

\begin{abstract}
We have studied the effect of interchain interaction on thermally excited solitons in a chargedensity wave for a Peierls system of commensurability 3. In such a system solitons with charges $\pm 2 e / 3$ are expected. It is shown that the interchain coupling in some cases will generate solitons with lower and higher charge than $\pm 2 e / 3$. The effect of discreteness is taken into account and gives rise to chaotic deformed solitons as the interchain coupling increases. The model may be applied to tetrathiafulvalene tetracyanoquinodimethane (TTF-TCNQ) under 19-kbar pressure.
\end{abstract}

There has been considerable interest in studying elementary nonlinear excitations (solitons) in coupled electron-phonon models for one-dimensional conductors, such as polyacetylene. ${ }^{1,2}$ In particular, it has been shown ${ }^{3}$ that solitons in systems near commensurability carry charges given by fractions of the elementary charge $e$. Attention has been drawn to the analogy with relativistic field theory models, where states with fractional fermion charge also appear. ${ }^{4}$

In this Communication we investigate numerically fractionally charged solitons in a phenomenological charge-density-wave (CDW) model for a Peierls system of commensurability 3 . In such system it is expected that the solitons (or " $\phi$ particles") carry charges $\pm 2 e / 3$. $^{5}$ However, we shall here also take into account the Coulomb interaction between neighboring chains. We find situations where this coupling generates two types of solitons with different charges. With increase of the interaction, which corresponds to lowering the temperature, the charges of the solitons change: The charge of one type of solitons increases, the charge of another type decreases. In this process the charges take real values less than $2 e$. When the soliton width is comparable to the lattice spacing, the discreteness will influence the solitons and deform them in a "chaotic" way. The model may in some temperature intervals be applicable to the organic conductor tetrathiafulvalene tetracyanoquinodimethane (TTF-TCNQ), which under 19-kbar pressure has commensurability 3 in the $b$ direction. ${ }^{6,7}$

The charge-density wave is of the form $\rho(x)=\psi \exp \left[i\left(q_{0} x+\tilde{\phi}(x)\right]\right.$. We assume that the amplitude $\psi$ does not depend on $x$, but we allow for local deformations in the phase $\tilde{\phi} . \quad q_{0}=2 k_{F}$ is the wave vector for the CDW and the associated Peierls distortion. Due to the commensurability of 3 the
$\mathrm{CDW}$ has a threefold-degenerate ground state, and the effective commensurability potential for the phase $\tilde{\phi}$ is taken to be of the form

$$
V_{c}(\tilde{\phi})=V_{0}(1-\cos 3 \tilde{\phi}) \quad\left(V_{0}>0\right),
$$

with an effective coupling constant $V_{0}$ proportional to $\psi^{3}$.

The physical system behind the model is a transfer salt with two different types of chains. We assume that the sublattice of one chain type orders first and study the CDW with phase $\tilde{\phi}$ on one of the chains of the other type, as it starts to order.

For TTF-TCNQ one would expect that there is a coupling between $\psi$ and the ordering period in the $a$ direction perpendicular to the chains, as found at normal pressure. ${ }^{8}$ From experiments at $19 \mathrm{kbar}$ it is known that this period is one lattice unit at low temperatures. $^{7}$ In that case the neighboring CDW's have the same phase $\eta$ (Fig. 1). The dominating contribution to the interchain coupling is the Coulomb interaction, which is restricted to neighboring chains. Coulomb interaction tends to bring neighboring CDW's in antiphase

$$
V_{\text {in }}(\tilde{\phi})=2 U_{0} \cos (\tilde{\phi}-\eta) \quad\left(U_{0}>0\right) .
$$

$U_{0}$ is an effective interchain coupling proportional to $\psi$. $V_{\text {in }}(\tilde{\phi})$ has periodicity $2 \pi$ in $\tilde{\phi}$. We assume that the phase $\eta$ is at a lock-in (commensurate) value 0 , $2 \pi / 3,4 \pi / 3$ and express the potentials in the variable $\phi=\tilde{\phi}-\eta$ :

$$
V(\phi)=V_{0}(1-\cos 3 \phi)+2 U_{0} \cos \phi .
$$

The potential ${ }^{9}(3)$ is symmetric around $\phi=\pi$, but becomes asymmetric when $\eta$ is not commensurate. In such cases the solitons have integer charges $\pm 2 e .^{10}$ 


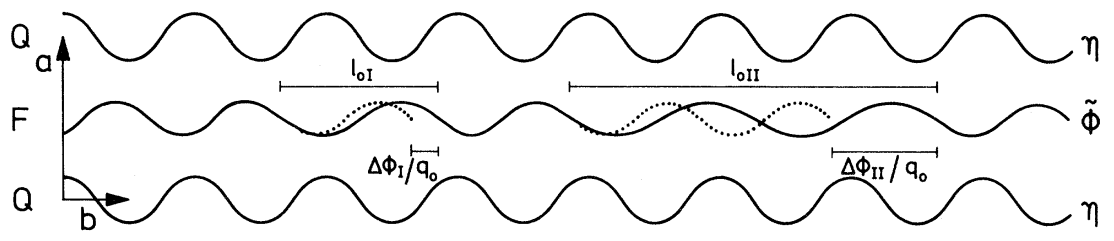

FIG. 1. Soliton excitation on a period three CDW with phase $\tilde{\phi}$. The CDW interacts with the neighboring CDW's (phase $\eta$ ). $l_{0 \mathrm{I}}$ and $l_{0 \mathrm{II}}$ are the widths of the type-I and -II solitons; $\Delta \phi_{\mathrm{I}}$ and $\Delta \phi_{\mathrm{II}}$ are their phase shifts. $F$ and $Q$ refer to the different chains in TTF-TCNQ.

The discreteness of the lattice is taken into account following Aubry ${ }^{11}$ and Bak and Pokrovsky. ${ }^{12}$ By defining $\phi_{n}$ as the CDW phase on the $n$th site of the chain and including the local strain energy, the potential energy on one chain becomes

$$
\begin{aligned}
H= & \sum_{n=-\infty}^{\infty} \lambda\left(\phi_{n+1}-\phi_{n}\right)^{2}+V_{0}\left(1-\cos 3 \phi_{n}\right) \\
& +2 U_{0} \cos \phi_{n} .
\end{aligned}
$$

The static excitations in $\phi_{n}$ (" $\phi_{n}$ particles") $)$ which minimize the energy are found by differentiating (4) with respect to $\phi_{n}$

$$
\begin{aligned}
& W_{n+1}=W_{n}+A \sin 3 \phi_{n}-B \sin \phi_{n}, \\
& \phi_{n+1}=\phi_{n}+W_{n+1},
\end{aligned}
$$

where $A=3 V_{0} / 2 \lambda, B=U_{0} / \lambda$ and the phase difference is defined by the last equation. These equations are a two-dimensional discrete area-preserving mapping depending on two parameters $A$ and $B$, which are proportional to the coupling constants $V_{0}$ and $U_{0}$, respectively.

We investigate the $\phi_{n}$ excitations within a "phasespace" plot $\left(\phi_{n}, W_{n}\right)$, which can be generated by iterations of the mapping (5) with different initial points. In the continuum limit (5) turns into the equation $\phi_{x x}=A \sin 3 \phi-B \sin \phi$, which is the triple sine-Gordon equation without a kinetic term. The triple sine-Gordon equation is not a integrable system, but numerical investigations have shown the existence of two different solitons (kinks). ${ }^{13}$

The potential $V(\phi)=V_{0}(1-\cos 3 \phi)+2 U_{0} \cos \phi$ has extrema for $\phi=0, \pi$, and

$$
\phi=\arcsin \left[ \pm\left(\frac{3}{4}-\frac{U_{0}}{6 V_{0}}\right)^{1 / 2}\right](\bmod 2 \pi)
$$

When there is no interchain coupling $\left(U_{0}=0\right)$ the mapping (5) will connect the ground states $(0,2 \pi / 3,4 \pi / 3)$ by solitons of the same type with phase shift $2 \pi / 3$. However, when the interchain coupling is nonzero the ground-state degeneracy becomes twofold and two types of solitons appear, a usual sine-Gordon kink (type I) and another kink with larger phase shift (type II). The physical picture of the kinks is shown schematically on Fig. 1. The kinks stretch (antikinks contract) the CDW over a finite number of lattice sites and may be considered as defects in the CDW. In a dynamical description these charged kinks account for the conductivity when sliding of the CDW is suppressed by commensurability pinning.

Figure 2 shows the potential and a phase-space
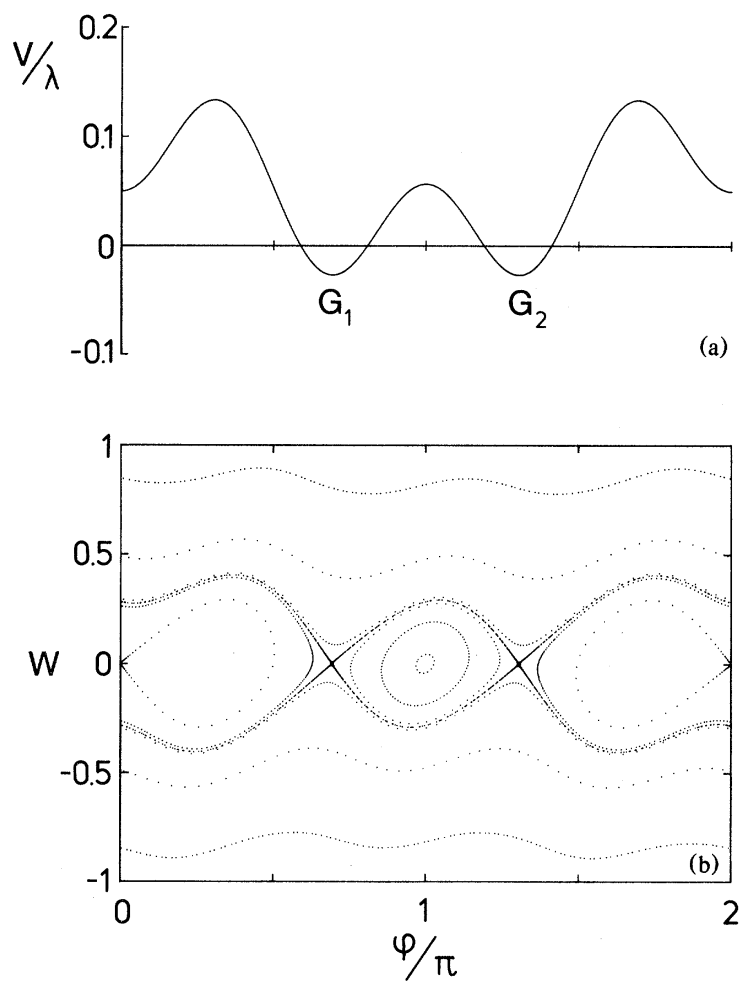

FIG. 2. (a): The pinning potential [Eq. (3)] and (b): phase-space plot $\left(\phi_{n}, W_{n}\right)$ for $A=0.08$ and $B=0.025$. The relative strength of the coupling constants is $U_{0} / V_{0}=0.46$. The curves between the ground states $\left(G_{1}\right.$ and $\left.G_{2}\right)$ consist of discrete points and are generated by many iterations. A soliton (or antisoliton) is created by following this iteration from one ground state to the other. These curves are weakly chaotic. The other curves are invariant, regular trajectories. Each curve is generated from one initial point. 
plot $(\bmod 2 \pi)$ for $A=0.08$ and $B=0.025$ with $U_{0} / V_{0}=0.46$. Clearly there are two types of kinks running between the ground states $\left(G_{1}\right.$ and $\left.G_{2}\right)$ in the upper half of the phase space, whereas the corresponding antikinks are in the lower part. The kinks are generated by discrete points, and their width are numerically found to be $l_{0 \mathrm{I}} \sim 20$ lattice spacings and $l_{0 I I} \sim 24$, which are of the order of magnitude estimated for TTF-TCNQ. ${ }^{5}$ The curves in the phase space near the ground states describe soliton lattices, which on an infinite chain have infinite excitation energy.

The charges of the solitons are calculated as follows. The charge transfer $\rho$ between two different chains determines the period $\lambda_{2 k_{F}}$ of the CDW, $\lambda_{2 k_{F}}=2 b / \rho$, and the density of condensed electrons in the CDW, $n_{s}=\rho / b$. Changes in the phase $\phi_{n}$ imply a local modulation in $n_{s}$, which in the continuum limit is given by ${ }^{5} n_{s}(x)=n_{s} q_{0}^{-1} \nabla_{x} \phi(x)$. The charge carried by a soliton is obtained by integration over the phase gradient

$$
e^{*}=-e n_{s} q_{0}^{-1} \int \nabla_{x} \phi d x=-e n_{s} q_{0}^{-1} \Delta \phi .
$$

Equation (6) determines the extrema of the potential, and the phase shift $\Delta \phi$ between the ground states is easily obtained as a function of $U_{0} / V_{0}$. The charges of the solitons on Fig. 2 are for type I

$$
e_{\mathrm{I}}^{*} / e=-0.61 \ldots,
$$

and for type II

$$
e_{\mathrm{II}}^{*} / e=-1.38 \ldots .
$$

The corresponding antisolitons have charges of the opposite sign. With further increase of interchain coupling, which corresponds to lowering the temperature, the type-I and -II solitons exhibit shorter and longer phase shift, respectively. That is, the charges pass continuously all real values; type I gets lower charge, type II higher charge ( $2 e$ is the highest value).

Figure 3 shows a phase-space plot for $U_{0} / V_{0}=3$. The charges of the solitons are in this case calculated to $e_{\mathrm{I}}^{*} / e=-\frac{1}{3}, e_{\mathrm{II}}^{*} / e=-1 \frac{2}{3}$. The width of the solitons has now decreased to $l_{0 \mathrm{I}} \sim 14$ and $l_{0 \mathrm{II}} \sim 9$ lattice spacings, and the effect of discreteness has indeed become very pronounced. In both phase-space plots (Figs. 2 and 3) the solitons are located in a chaotic regime. However, whereas the chaotic regime of Fig. 2 is very small and almost invisible, it has clearly broadened with increase of the interchain coupling (Fig. 3). Physically, the chaotic regime indicates that the lattice deforms the solitons, so that two soliton excitations of the same type and width at different places of the chain usually have different shapes. Whereas the type-II soliton in Fig. 3 is very chaotic the low-charged type-I soliton is still rather regular.

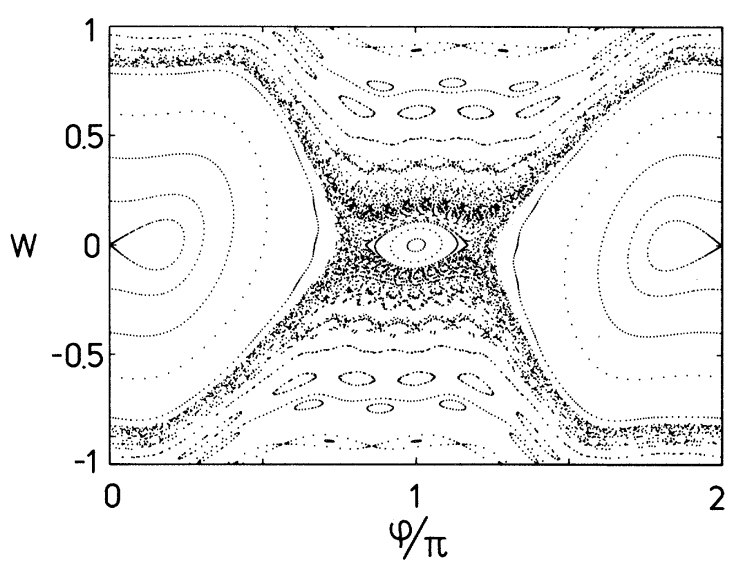

FIG. 3. Phase-space plot $\left(\phi_{n}, W_{n}\right)$ for $A=0.1$ and $B=0.2$, with $U_{0} / V_{0}=3$. The curves between the ground states has broadened compared to Fig. 2, and the solitons show clear chaotic behavior. This chaotic regime is generated from one initial point.

This is because the width of the type-II soliton is smaller than the width of the type-I soliton.

The strengths of the commensurability and interchain couplings have not yet been calculated as a function of temperature and pressure for TTFTCNQ. Probably the strength of the effective interchain coupling used in Fig. 3 is overestimated. However, our goal is to describe qualitatively the effect of interchain interaction and discreteness on the solitons. The excitation energy is smaller for the lowcharged than for the high-charged soliton. Experimentally one should therefore observe a net decrease in the charge of the current carrying solitons, as the temperature is lowered below the sublattice ordering temperature.

In conclusion, we have shown that the interchain coupling can affect the charges of soliton excitations in the charge-density wave for a Peierls system of commensurability 3 . With increase of interchain interaction the charges move continuously away from the commensurate values $\pm 2 e / 3$ and $\pm 4 e / 3$.

After this work was completed we learned about an unpublished work by M. J. Rice and J. Timonen ${ }^{10}$ on a continuum version of a model similar to the one presented here. Their results are in agreement with ours, but these authors also discuss situations in which the interchain coupling will destroy the fractionalization of charge, which corresponds to cases where $\eta$ does not have commensurate values. We are grateful to J. Timonen for informing us about this work. Also, we are indebted to Per Bak for suggesting corrections to the original version of this manuscript and to P. Cvitanović and P. L. Christiansen for valuable discussions. This work has been supported in part by the Danish Natural Science Research Council. 
*Present address.

${ }^{\dagger}$ Present address: Center for Nonlinear Studies, Los Alamos National Laboratory, Los Alamos, N.M. 87545.

${ }^{1}$ W. P. Su, J. R. Schrieffer, and A. J. Heeger, Phys. Rev. Lett. 42, 1698 (1979); Phys. Rev. B 22, 2099 (1980).

${ }^{2}$ M. J. Rice, Phys. Lett. 71A, 152 (1979).

${ }^{3}$ W. P. Su and J. R. Schrieffer, Phys. Rev. Lett. 46, 738 (1981).

${ }^{4}$ D. K. Campbell and A. R. Bishop, Phys. Rev. B 24, 4859 (1981); R. Jackiw and J. R. Schrieffer, Nucl. Phys. B190, [FS3], 253 (1981).

${ }^{5}$ M. J. Rice, A. R. Bishop, J. A. Krumhansl, and S. E. Trullinger, Phys. Rev. Lett. 36, 432 (1976).

${ }^{6}$ R. H. Friend, M. Miljak, and D. Jérome, Phys. Rev. Lett. 40, 1048 (1978).

${ }^{7}$ S. Megtert, R. Comés, C. Vettier, R. Pynn, and A. F. Gari- to, Solid State Commun. 37, 875 (1981).

${ }^{8}$ P. Bak and V. J. Emery, Phys. Rev. Lett. 36, 978 (1976).

${ }^{9}$ This potential is also applicable when the perpendicular period is not one lattice unit. If one for the neighboring phases $\eta_{1}, \eta_{2}$ assume that $\left(\eta_{1}+\eta_{2}\right) / 2$ is a commensurate value we again obtain (3) with a modified coupling constant $U_{0}^{\prime}$.

${ }^{10} \mathrm{M}$. J. Rice and J. Timonen, Phys. Lett. (in press).

${ }^{11} \mathrm{~S}$. Aubry, in Solitons and Condensed Matter Physics, edited by A. R. Bishop and T. Schneider (Springer, Berlin, 1978), p. 264.

12P. Bak, Phys. Rev. Lett. 46, 791 (1981); P. Bak and V. L. Pokrovsky, ibid. 47, 958 (1981).

${ }^{13} \mathrm{O}$. H. Olsen and M. R. Samuelsen, Wave Motion $\underline{4}, 29$ (1982). 